node, and $\mathrm{D}$ is the number of drawing moves. Provided that at least one node in the tree has a score of -1 , even if due only to the opponent's blunder, a search of the resulting 7-ply tree will provide a greater practical chance of success against an imperfect opponent than will selecting a drawing root move at random.

Various refinements to this concept suggest themselves, but are beyond the scope of this note. Although the application of this concept to chess-endgame databases is new, so far as I am aware, the idea of tree search assuming imperfect play by the opponent has been described earlier (e.g., Michie, 1981). In other words, when desperate, it is good tactics to mislead one's opponent. The opponent, if not omniscient, i.e., if a human being rather than a computer database, is therefore classified as being, in some sense, contemptible. The notion of a contempt factor has been broached in literature by Slate and Atkin (1977) for full games. Without any risk (as opposed to its use in full games) it may now be applied in endgames tabulated in a database.

\title{
References
}

Michie, D. (1981). A theory of evaluative comments in chess with a note on minimaxing. The Computer Journal, Vol. 24, No. 3.

Slate, D.J. and Atkin, L.R. (1977). CHESS 4.5 - The Northwestern University Chess Program. Chess Skill in Man and Machine (Ed. P.W. Frey), pp. 82 - 118. Springer Verlag, New York.

\section{CORRECTING GRANDMASTERS' ANALYSES IN ELEMENTARY ENDGAMES}

\section{Rasmussen}

Tranbjerg, Denmark

\section{Editor's Introduction}

It may be challenging to our readers to analyse this note by Lars Rasmussen dealing with the KRKN endgame, the very same subject that has been treated earlier in this issue by Denis Verhoef and Jacco Wesselius. It will be remarked that the two authors' approaches are as distinct as they could be; nevertheless they are complementary. Whereas Verhoef and Wesselius give an a priori, classificatory treatment, Rasmussen below provides an a posteriori, database analysis. It is not the least of Rasmussen's merits that a home computer sufficed to hold the database and the program to consult it.

Recently, I have undertaken a definitive analysis of some technical endgames by computer, the most interesting of which are Queen versus Rook, and Rook versus Knight. I have compared my computer's analysis with the existing endgame theory and have found some interesting results as the following KRKN examples will show.

\section{The first instance}

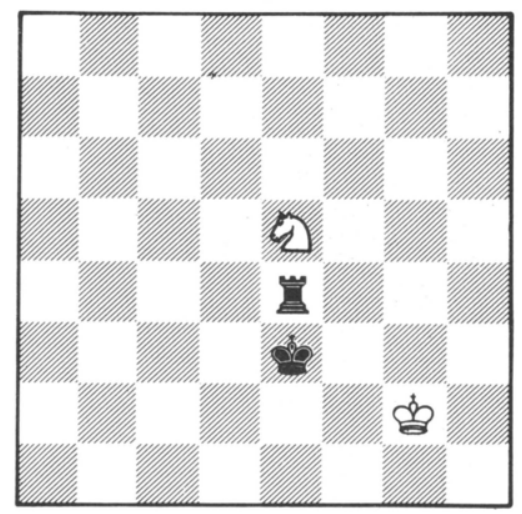

The position of Diagram 1 arose in the game Gosh - Gipslis (Calcutta 1979). White played 1. Nf7 and lost. Gipslis means according to ECE (rook endings II, pos. 393) that White could make a draw by 1 . Nd7! Kf4 2. Nc5! This is not true, because after 1. Nd7 Kf4 2. Nc5 will follow 2. ... Re2! Now two variations are possible (A) 3. Kf1 and (B) 3. Kh3.
White: $\mathrm{Kg} 2 \mathrm{Ne} 5$

Black: Ke3 Re4

Black to move. 
Variation A reads as follows:

\section{Kf1 Rc2 4. Ne6}

1) 4. Nd3 Ke3 5. Ne5 Rc5 6. Nd7 (6. Ng4 Kf3 7. Nh2 Kg3) Rg5! 7. Nb6 Kd4 8. Ke2 Rg7!

2) 4. Nb3 Ke3 5. Na5 Rc5 6. Nb7 Rd5

3) 4. $\mathrm{Na6} \mathrm{Ke} 4$ 5. Nb8 Kd5 6. Nd7 Rc6!

4. ... Ke3! 5. Ng5

Also losing is 5. Kg1 Rc6 6. Nd8 Rg6 7. Kf1 Kf3 8. Ke1 Rf6 9. Nb7 Rf5 10. Kd2 Rb5

5. ... Rf2 6. Kg1 Rf5 7. Ne6

Another knight move does not help either: 7. Nh3 Kf3 8. Nf2 Rd5 9. Nh3 Rd1 10. Kh2 Rd2 11. Kh1 Kg3 12. Ng1 Rh2 \#

7. ... $\operatorname{Re} 5$ 8. Nd8

Two variations are
1) 8. Nc7 Kf3 9. Na6 Rg5 10. Kh2 Rg2 11. Kh1 Kg3!
2) 8. Nf8 Re7! 9. Kh2 Ke4 10. Ng6 Rh7
8. ... Ke4 9. Nc6
9. Nf7 Rd5 10. Kg2 Kf4
9. ... Rc5 10. Nb4 Rc4 11. Na6 Kd5 12. Kf2 Kd6 13. Ke3 Ra4

Variation B reads:

3. Kh3 Kf5! (3. ... Rc2? 4. Ne6= ) 4. Kg3!
1) 4. Nb7 Rc2! 5. Nd6 Kf4! 6. Nb5 Ke5 7. Kg4 Rb2 8. Nc7 Rb7
2) 4. Kh4 Rb2! 5. Nd3 Rc2 6. Ne1 Re2 7. Nf3 Kf4
4. ... Rc2! 5. Na4 Ke4 6. Kg4 Rc6 7. Nb2 Ke3! (7. ... Kd4? 8. Nd1 Rc2 9. Kf3 = )
8. Kf5! Kd4!
(8. ... Kd2? 9. Ke5 Kc3 10. Kd5 Rc8 11. Na4 Kb4 12. Nb6 =)
9. Kf4 Re1! 10. Na4 Rb1

The second instance

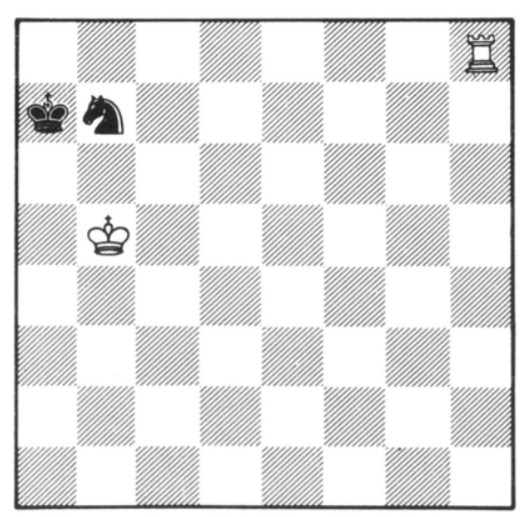

DIAGRAM 2
Diagram 2 has been taken from Euwes "Endspieltheorie und -praxis" pos. 78. Euwe claims that 1. Rh7 leads to a draw after 1. ... Kb8 2. Kb6 Nd8. But White plays 2. Kc6! Na5 3. Kb6 Nc4 4. Kb5 Nd6 5. Kc6 Nc4 6. Rb7 Kc8 7. Rb5 Kd8 8. Rc5 Ne3 9. Rg5 Nc4 10. Kc5 Na5 11. Kb5 +-, or 2. ... Nd8 3. Kd7 Nb7 4. Rh5 Ka7 5. Kc8 Nd6 6. Kc7 Ne8 7. Kc6 Nf6 8. Rh4 Kb8 9. Rf4 Nh5 10. Rf7 Ng3 11. Rb7 $\mathrm{Ka8} 12 . \mathrm{Kc7}+-$. 
A maximin position

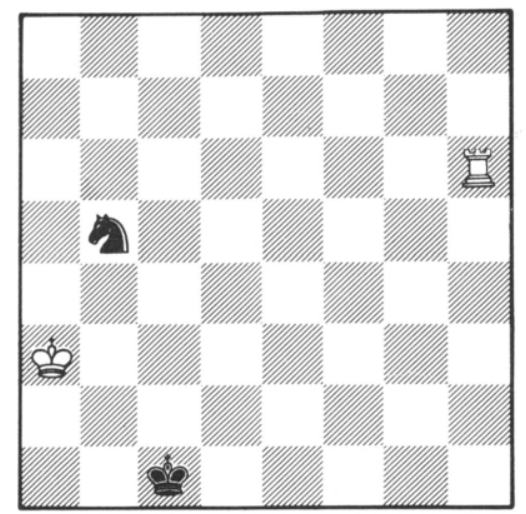

DIAGRAM 3
The position of Diagram 3 is one of exactly two positions (ignoring symmetries) in which it takes a maximum of 27 moves to capture the Knight. (The other one is $\mathrm{Ka} 4, \mathrm{Ra} 8$ $\mathrm{Ka} 2, \mathrm{Nd} 7$.) An optimal variation is presented below.

1. Kb4 Nd4 2. Kc3 Ne2 3. Kd3 Nf4 4. Ke3 Ng2 (4... Nd5 5. Kd4 Nb4 6. Kc3 Nd5 7. Kc4 Ne3 8. Kd3 Nd5 9. Rh4 Kb2 10. Rd4! +-) 5. Kf2 Nf4 6. Rd6! Kc2 7. Ke3! Ng2 8. Kf3 Nh4 9. Ke2!! (with the idea Rf6, Kf2-g3-h4) 9. Nf5 10. Rc6 Kb3 11. Kd3 Kb4 12. Re6! Kb3 13. Rb6 Ka4 14. Kc4 Ne3 15. Kd4 Nc2 (15. ... Nf5 16. Kc5! Ne3 17. Rb2! Ka3 18. Rd2! +-; 15. ... Ka5 16. Rb2! +-) 16. Kc3 Ka5 17. Rb2! Ne3 18. Kd4 Nf5 19. Kc5 Ka6 20. Rf2 Ng3 21. Kd5 Kb6 22. Ke5 Kc5 23. Rg2 Nf1 24. Rc2 Kb4 25. Kf4 Kb3 26. Rf2 +-
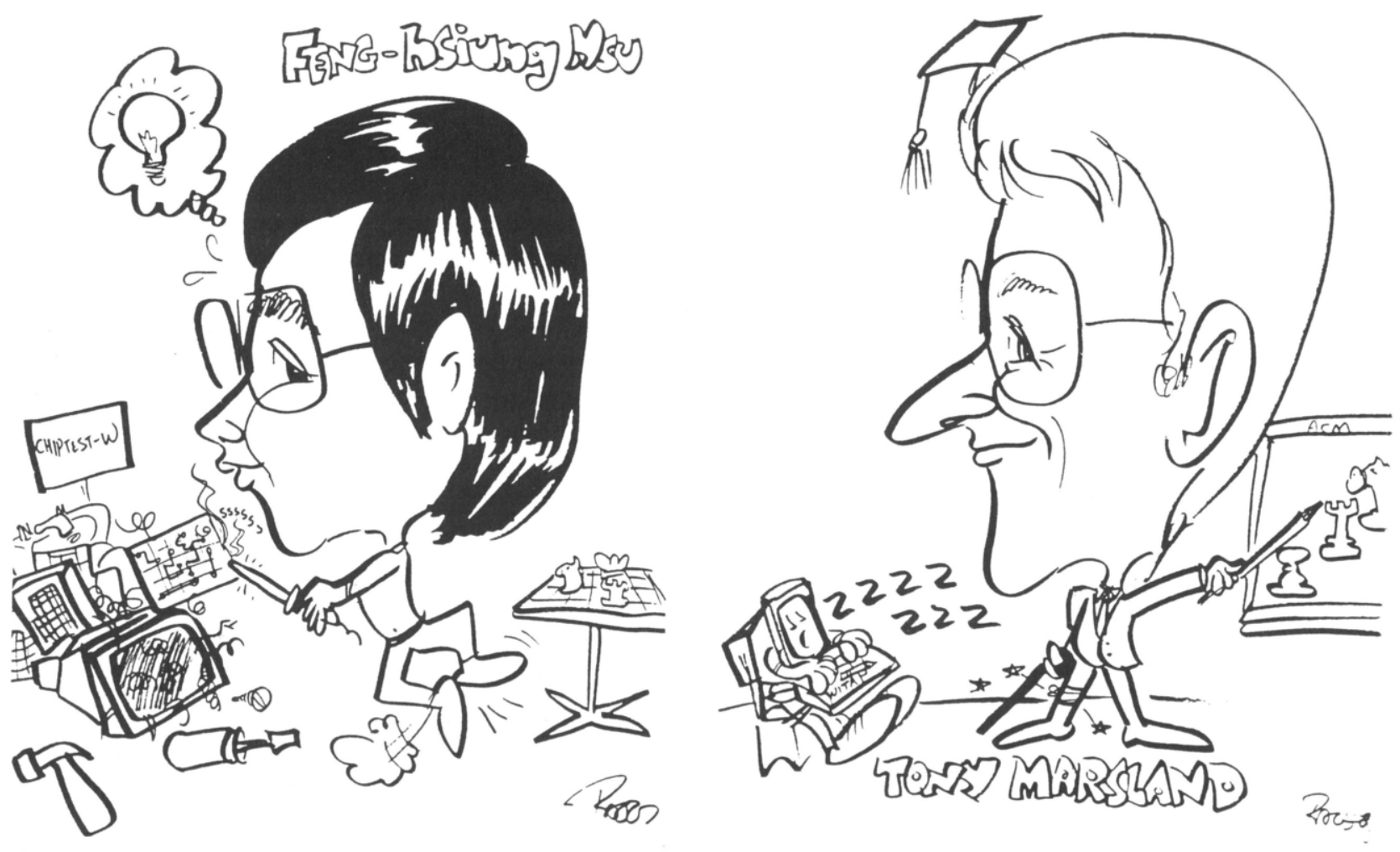

Cartoons by Jeff Ragsdale 